\title{
Enhancement of Rabi Splitting in a Microcavity with an Embedded Superlattice
}

\author{
J. H. Dickerson* and E. E. Mendez \\ Department of Physics and Astronomy, State University of New York at Stony Brook, Stony Brook, NY 11794-3800 \\ A. A. Allerman \\ Sandia National Laboratory, Albuquerque, NM 87185 \\ S. Manotas, F. Agulló-Rueda, and C. Pecharromán \\ Instituto de Ciencia de Materiales, CSIC, Cantoblanco, 28049 Madrid, Spain
}

(November 2, 2018)

\begin{abstract}
We have observed a large coupling between the excitonic and photonic modes of an AlAs/AlGaAs microcavity filled with an $84-\AA / 20 \AA$ GaAs/AlGaAs superlattice. Reflectivity measurements on the coupled cavity-superlattice system in the presence of a moderate electric field yielded a Rabi splitting of $9.5 \mathrm{meV}$ at $\mathrm{T}=238 \mathrm{~K}$. This splitting is almost $50 \%$ larger than that found in comparable microcavities with quantum wells placed at the antinodes only. We explain the enhancement by the larger density of optical absorbers in the superlattice, combined with the quasi-two-dimensional binding energy of field-localized excitons.
\end{abstract}

73.21.Cd,78.30.Fs,78.40.Fy

The ability of semiconductor multilayers to mimic atomic cavities has allowed the observation of phenomena that until recently seemed reserved to atomic physics. Among these, the best studied so far is the coupling of a two-level atom and the electromagnetic modes of a cavity enclosing it, which results in the so-called Rabi splitting when there is a resonance between one of those modes and the energy difference between the atomic levels. 1 Analogously, in a semiconductor microcavity formed by two dielectric mirrors and a quantum well (or a small set of quantum wells) in the cavity between them, the excitonic mode of the quantum well is coupled to a photonic mode of the cavity when the characteristif energies of the two modes, $\mathrm{h} \nu_{\text {exe }}$ and $\mathrm{h} \nu_{\text {cav }}$, coincide. $\mathrm{a}$ (4)

If strong enough, this coupling is manifested as a splitting into two of the optical features associated with that energy, for instance, a minimum in the reflectivity spectrum or a peak in the absorption or luminescence spectrum. In atomic systems the Rabi splitting is seen easily at room temperature, but in semiconductors the splitting is much less apparent because of a smaller splitting-tolinewidth ratio and a considerable decrease with increasing temperature.

The magnitude of the splitting is proportional to the overlap between the exciton's wavefunction and the photon's electric field and to the square root of the prodyct of oscillator strength and linear density of excitons. 5 Therefore, it is not surprising that a considerable effort has been made to increase these factors. To this end, more than one quantum well has been placed at the antinodes of the mirror-defined cavity, and various material systems have been used. In microcavities made out of III-V semiconductors, such as GaAs, AlAs, and InAs, typical Rabi splittings are 6 to $7 \mathrm{meV}$ at low temperature and less than $5 \mathrm{meV}$ at room temperature. 58 Because of their larger exciton binding energy (and corresponding larger oscillator strength) CdTe-based microcavities have shown splittings as large as $23 \mathrm{meV}$ at $4 \mathrm{~K}$ and $17 \mathrm{meV}$ at $300 \mathrm{~K} .0$

The Rabi splitting is often limited by the imperfect confinement of the photon field within the physical boundaries of the cavity, which reduces the photonexciton overlap.

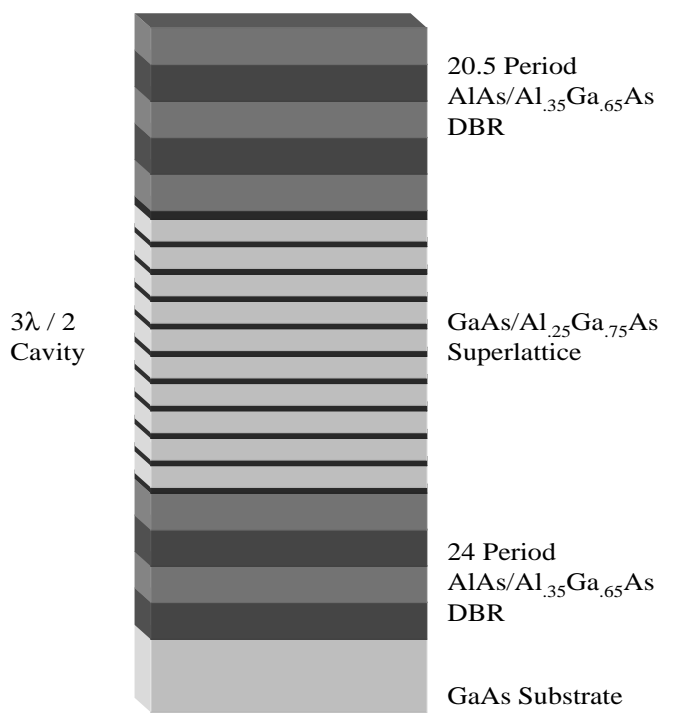

FIG. 1. Two Distributed Bragg reflector mirrors (DBRs), comprised of alternating $\frac{\lambda}{4}$ layers of $689 \AA$ AlAs and $602 \AA$ $\mathrm{Al}_{.35} \mathrm{Ga}_{65} \mathrm{As}$, flanking an $84 \AA \mathrm{GaAs} / 20 \AA \mathrm{Al}_{.25} \mathrm{Ga} .75 \mathrm{As}$ superlattice that defines a $\frac{3 \lambda}{2}$ cavity. The DBRs were either undoped (Sample B) or doped (Sample A) p-type $\left(1 \times 10^{18}\right.$ of $\mathrm{Be})$, for the top mirror, and n-type $\left(1 \times 10^{18}\right.$ of $\left.\mathrm{Si}\right)$ for the bottom mirror. 
To

ameliorate

that

effect, selectively oxidized AlAs/GaAs mirrors, with larger index-of-refraction contrast, have been used. 11, 12 Others have embedded quantum wells_in the mirror regions, thus increasing that overlap. 13 In still another scheme the entire cavity between the two mirrors consisted of just one material (GaAs), thus forming a "bulk" microcavity in which the larger number of excitons per unit length partially compensated both for the smaller binding energy of three-dimensional (3D) excitons and the spatially non-uniform coupling to the photon field. 14 The Rabi splitting found experimentally for these structures was about half of that in comparable quantum-well microcavities.

In this Communication we present a simple way of enhancing the exciton-carvity coupling in a quantum-well microcavity system. 15 By exploiting the localization properties of a superlattice under a longitudinal electric field, we have overcome the limitation in a previous scheme brought out by the small binding energy of a 3D exciton, while keeping the benefits of a thick active medium. Our approach consists of filling the entire cavity with a superlattice subjected to an electric field, rather than placing a few isolated wells at the antinodes of the cavity. A narrow-barrier superlattice within the active region maximizes the density of quantum wells while the field enhances the exciton binding energy threysh Wannier-Stark localization of the superlattice states 16,47 Using this scheme we have found a $9.5 \mathrm{meV}$ Rabi splitting at $\mathrm{T}=238 \mathrm{~K}$, which represents almost a $50 \%$ increase of the splitting found in a comparable optical structure in which the field is absent and quantum wells are placed exclusively at the antinodes.

In a superlattice of period D, the electronic wavefunctions are extended, and the exciton's binding energy is comparable to that of a 3D semiconductor. However, under a longitudinal (that is, parallel to the superlattice direction) electric field $\varepsilon$, the spatial extension of the wavefunctions is reduced to a length of the order of $\frac{\Delta}{e \varepsilon}$, where $\Delta$ is the energy width of the miniband, and e is the electronic charge. Beyond a certain field, electrons and holes become localized into individual quantum wells, excitons become quasi two-dimensional, and their binding energy increases drastically. Then, it should be possible to achieve enhanced Rabi splitting by filling a semiconductor microcavity with a superlattice that is subjected to a strong enough electric field, applied either internally or externally.

The microcavities we have used to test this idea consisted of two distributed Bragg reflector mirrors (DBRs) flanking a 30 period $84-\AA$ GaAs / $20-\AA \mathrm{Al}_{.25} \mathrm{Ga} .75 \mathrm{As}$ superlattice, which formed the active region of the cavity (see Fig. 1). The top (bottom) reflector had 20.5 (24) periods of alternating $689-\AA \mathrm{AlAs}$ and $602-\AA \mathrm{Al} .{ }_{35} \mathrm{Ga} .65 \mathrm{As}$ $\frac{\lambda}{4}$ layers. In sample $\mathrm{A}$, the top mirror was p-type doped (Be,1 times $10^{18} \mathrm{~cm}^{-3}$ and the bottom mirror was doped n-type ( $\mathrm{Si}, 1$ times $10^{18} \mathrm{~cm}^{-3}$, while the cavity was un- doped, thus forming a $\mathrm{p}-\mathrm{i}-\mathrm{n}$ heterostructure. In sample $\mathrm{B}$, the entire structure was undoped. The number of superlattice periods was chosen so that the length of the cavity was $\frac{3 \lambda}{2}$, where $\lambda=\frac{\lambda_{0}}{n}$. $\lambda_{0}$ is the wavelength associated with the exciton binding energy $E_{e x}\left(\lambda_{0}=\frac{c h}{E_{e x}}\right) ; \mathrm{n}$ is the active region's index of refraction. c is the speed of light in vacuum, and $\mathrm{h}$ is Planck's constant. In our case, $\lambda_{0}$ equaled $8040 \AA$, which corresponds to the e1hh1 exciton energy for the $84 \AA / 20 \AA$ superlattice at around liquid-nitrogen temperature. We also prepared a quantum-well microcavity (sample $\mathrm{C}$ ) in which the superlattice of sample A was replaced by two uncoupled 89 $\AA$ quantum wells at each of the three anti-nodes of the $\frac{3 \lambda}{2}$ cavity. The focus of the work reported here is on sample A, with samples B and C serving as controls.

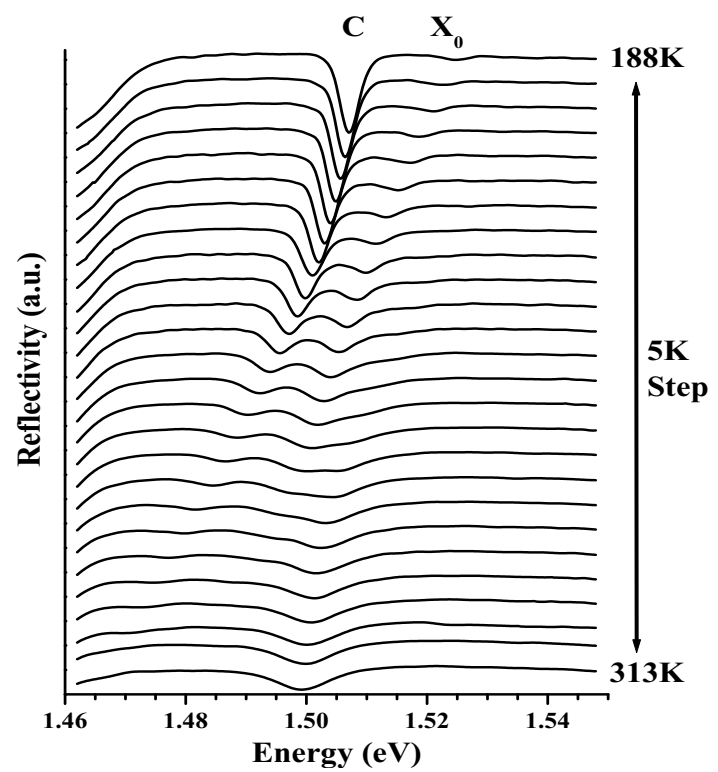

FIG. 2. Experimental reflectivity spectra for the doped microcavity (sample A in the text) across the $188 \mathrm{~K}$ to $313 \mathrm{~K}$ temperature range, in $5 \mathrm{~K}$ intervals. For clarity, the spectra have been shifted vertically in a cascade format. Two minima are easily observed at most temperatures, while an additional, very weak minimum can be discerned at some temperatures.

The Rabi splitting was determined primarily from reflectivity measurements, complemented by photoluminescence experiments. The energy coupling between the exciton and the cavity-photon modes was tuned in and out of resonance by varying either the temperature of the sample or the spot that was probed. A slight taper of the microcavity, inherent to the epitaxial MOCVD growth process, shifted the energy of the cavity mode along the radial direction of the 3" GaAs wafers on which the multilayers were grown. The cavity mode shifted approximately $10 \mathrm{meV}$ (and in one sample up to $30 \mathrm{meV}$ ) from the center to the edge, while the exciton energy remained practically unchanged. On the other hand, the energy of 
the exciton changed with temperature at a rate of 3.8 meV per $10 \mathrm{~K}$, a rate more than five times larger than that of the cavity mode.

The reflectivity of the microcavities was measured at normal incidence, using a micro-reflectivity spectroscopy set-up coupled to a broad-spectrum quartz tungsten halogen lamp and a filter to block photon energies above 1.9 $\mathrm{eV}$. The spatial and spectral resolutions of the system are better than 2 mum and $0.20 \mathrm{meV}$, respectively.

Figure 2 shows reflectivity spectra for sample $\mathrm{A}$ in the temperature range $188 \mathrm{~K}-313 \mathrm{~K}$ in the $1.46 \mathrm{eV}-1.55 \mathrm{eV}$ spectral region, which corresponds to the high-reflectivity region of the Bragg reflectors. At the lowest temperature the strongest reflectivity minimum is at $1.5078 \mathrm{eV}$, with a second one at $1.5238 \mathrm{eV}$ and a third, barely visible dip at $1.5382 \mathrm{eV}$. As the temperature increases, the latter two minima shift to lower energy faster than the former, and simultaneously the relative intensity of the three minima gradually changes. Above $260 \mathrm{~K}$, the highest-energy minimum is most pronounced and shifts the least as the temperature increases.

The energy positions of the three minima as a function of temperature are summarized in Fig. 3. The linear shift of these features at the two ends of the temperature range attests their origin: at low $\mathrm{T}$, the stronger, lowenergy feature evidences the cavity mode (labeled $\mathrm{C}$ in the figure), whereas the two features at higher energy have excitonic character and are labeled as $X_{0}$ and $X_{1}$ in Fig. 3. At high $\mathrm{T}$, the situation is reversed: the stronger, high-energy minimum corresponds to the cavity mode and the much weaker minima at low energy are excitonic in nature. At intermediate temperatures, two anticrossings are observed, one between $\mathrm{C}$ and $X_{0}$ at $\mathrm{T}$ $=238 \mathrm{~K}$ and another between $\mathrm{C}$ and $X_{1}$ at $\mathrm{T}=253$ $\mathrm{K}$, with anticrossing energies of $9.5 \mathrm{meV}$ and $6.9 \mathrm{meV}$, respectively.

We interpret the $X_{0}$ line as the electric-field localized e1-hh1 exciton of the superlattice, which interacts with the cavity mode $\mathrm{C}$ and forms a mixed state at intermediate temperatures. The minimum $9.5 \mathrm{meV}$ splitting corresponds to the maximum coupling and is a measure of the Rabi splitting. This interpretation is supported by temperature-dependent photoluminescence measurements, which show a behavior and anticrossing similar to that of $X_{0}$ and $\mathrm{C}$ in Fig. 3. Room temperature, position-dependent reflectivity measurements on a different portion of sample A exhibited two clear minima whose relative strength and energy separation depend on the spot probed, from which a Rabi splitting of $8.5 \mathrm{meV}$ was inferred.

The $9.5 \mathrm{meV}$ splitting is almost $50 \%$ larger than the splitting obtained in similar temperature-dependence reflectivity measurements in sample $\mathrm{C}$, in which the superlattice was replaced by quantum wells at the antinodes. This shows that the larger number of wells favors a larger Rabi splitting, even though many are not in optimal positions in the cavity to couple effectively to the optical field. That the superlattice excitons are localized by the electric field is essential to the enhancement of Rabi splitting, as evidenced by the $4.5 \mathrm{meV}$ splitting obtained for sample B. In sample A, the built-in field of the p-i-n structure provides substantial, if not complete (see below), wavefunction localization for both electrons and holes. In contrast, the cavity in sample B is in flat-band configuration; the electrons and holes are delocalized and the exciton binding energy is comparable to that of bulk material.

The origin of the $X_{1}$ line in Fig. 3, the line responsible for a secondary anticrossing of $6.9 \mathrm{meV}$ with the cavity mode at $\mathrm{T}=253 \mathrm{~K}$, is less clear. One possibility is that $X_{1}$ is associated with the field-localized light-hole exciton (e1-lh1) of the superlattice. The calculated energy difference between the first heavy-hole and light-hole states in an isolated $84-\AA \mathrm{GaAs} / \mathrm{Al}_{.25} \mathrm{Ga}_{.75} \mathrm{As}$ quantum well is $18 \mathrm{meV}$, which is not very different from the $15 \mathrm{meV}$ average separation between the $X_{0}$ and $X_{1}$ lines when both are far from the anticrossing region with the cavity mode C. 18 Within this interpretation, the weakness of the reflectivity minima associated to $X_{1}$ (see Fig. 2) could be explained by the smaller oscillator strength of the light-hole exciton.

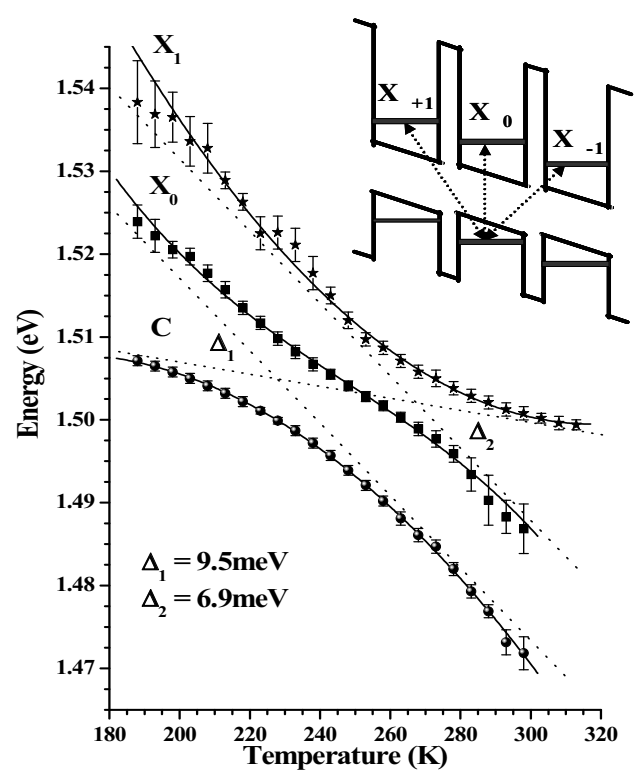

FIG. 3. Energies of the reflectivity minima in Fig. 2 plotted as a function of temperature. The two rapidly changing energies correspond to 0 and +1 heavy-hole exciton transitions of the Stark ladder ( $X_{0}$ and $X_{1}$, respectively) whereas the more gradually changing energy corresponds to the cavity mode, C. The dotted lines are guides to the eye to indicate the temperature trends of the uncoupled exciton and cavity modes.

Another possibility is that the $X_{1}$ line is due to a spatially indirect exciton involving electrons in one quantum well of the superlattice and holes in an adjacent well (see 
the inset of Fig. 3) that is, the +1 exciton involving the electron and hole Wannier-Stark ladders of the superlattice. Although at high electric fields $\left(\varepsilon>>\frac{\Delta}{e D}\right)$ there is complete localization of electrons and holes to individual wells, at smaller fields the localization is incomplete, with the electronic wavefunctions extended beyond a single well. In sample A, the calculated energy widths of the electron and heavy hole minibands are $19.5 \mathrm{meV}$ and $1.5 \mathrm{meV}$, respectively, which implies that the high-field regime is reached for $\varepsilon>>20 \mathrm{kV} / \mathrm{cm}$. If we assume that the built-in electric field in sample A's p-i-n configuration does not penetrate into the doped dielectric mirrors, then that field is $\approx 50 \mathrm{kV} / \mathrm{cm}$, which is sufficient for almost complete localization. However, in view of the wellknown difficulty to incorporate impurities as electrically active dopants in AlGaAs dielectric mirrors with high $\mathrm{Al}$ content, it is possible that the effective electric field across the superlattice in sample A is significantly smaller than $50 \mathrm{kV} / \mathrm{cm}$. Indeed, if we interpret the $X_{1}$ line as the +1 exciton, then the effective field is $15 \mathrm{kV} / \mathrm{cm}$, based on the $X_{0}-X_{1}$ energy separation.

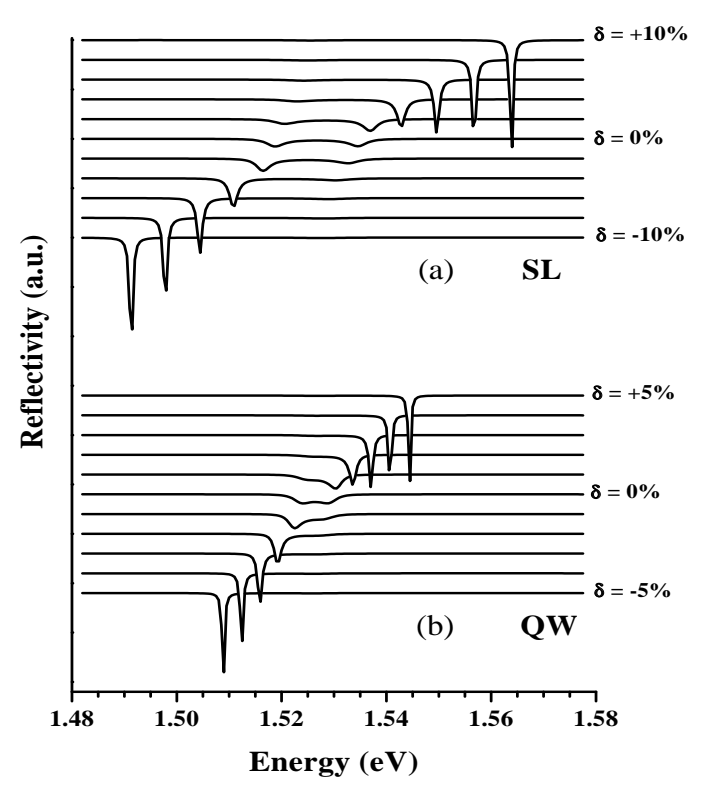

FIG. 4. Calculated reflectivity for a superlattice-cavity (a) and a quantum well-cavity (b) system. In the former, $30 \mathrm{GaAs}$ absorbers, each $80 \AA$ thick and separated from each other by $20 \AA$ of $\mathrm{Al}_{.25} \mathrm{Ga}_{.75} \mathrm{As}$, fill the cavity. In the latter, three $80 \AA$ GaAs absorbers are placed at the antinodes of the $\frac{3 \lambda}{2}$ cavity. The parameter $\delta$ represents the relative detuning between the pure exciton and cavity modes. In (a) a minimum splitting of $15 \mathrm{meV}$ between the two modes is obtained when $\delta=0$. In contrast, for (b) the corresponding splitting is only $4.5 \mathrm{meV}$.

In spite of the difference between these two field values, we favor the interpretation for $X_{1}$ in terms of spatially indirect excitons since it is consistent with the absence of the $X_{1}$ line in samples B and C. A light-hole exciton line would be present in the spectra of all three samples. The T-dependence PL spectra of sample A also favor that interpretation. In addition to a feature analogous to $X_{1}$, we resolved at low $\mathrm{T}$ a weak feature about 14 meV below that of $X_{0}$, which eventually had a small anticrossing with the cavity-mode peak. That feature can be seen as the -1 exciton of the Wannier-Stark ladder. The smaller anticrossing is then the result of the smaller binding energy of the \pm 1 exciton, in comparison with that of the $X_{0}$ exciton.

Our main observation-an enhanced Rabi-splitting in sample A between the spatially direct exciton and the cavity mode-agrees with a simple classical simulation of the reflectivity for a set of 30 GaAs absorbers (each 84 $\AA$ thick and $20 \AA$ apart from each other) distributed throughout the cavity defined by two dielectric mirrors. Figure 4 shows the calculated reflectivity spectra in the strong-coupling regime, for various values of the detuning parameter $\delta$, defined as $\delta=1-\frac{\nu_{e x c}}{\nu_{c a v}}$. For simplicity, we have kept constant the exciton energy $\mathrm{h} \nu_{\text {exc }}$ at $1.5098 \mathrm{eV}$ and varied the characteristic energy of the cavity, $\mathrm{h} \nu_{\text {cav }}$. We have used "realistic" values for the linewidth and oscillator strength of the exciton, $4 \mathrm{meV}$ and 0.035 respectively, so that the calculated lineshapes resemble the experimental curves at $238 \mathrm{~K}$. With this set of parameters the anticrossing is about $15 \mathrm{meV}$. In contrast, a similar simulation for a microcavity with only three 84 $\AA$-thick GaAs wells, each located at one of the antinodes of a $\frac{3 \lambda}{2}$ cavity, yielded a minimum separation three times smaller. The large difference in splitting between the two configurations confirms qualitatively our observations.

In this work, we have taken advantage of the builtin electric field of a p-i-n junction to illustrate how, using a simple scheme, an electric-field-localized superlattice embedded in a microcavity can enhance the excitonphoton coupling. Further enhancements are expected by applying a larger (external) electric field or by optimizing the well-to-barrier thickness ratio so that complete exciton localization is achieved. The ideas presented in this paper can also be combined with existing schemes (e.g. extending the superlattice into the mirrors) and/or implemented in other semiconductor materials (e.g., II-VI compounds) to obtain even larger Rabi splittings.

This work has been sponsored in part by the Army Research Office and by the Spanish Interministerial Commission for Science and Technology (CICyT). We acknowledge partial support from a Turner Fellowship (J. H. D.) and a Spanish CAM fellowship (S. M.).

${ }^{1}$ Y. Zhu, D. J. Gauthier, S. E. Morin, Q. Wu, H. J. Carmichael, and T. W. Mossberg, Phys. Rev. Lett. 64, 2499 (1990).

${ }^{2}$ C. Weisbuch, M. Nishioka, A. Ishikawa, and Y. Arakawa, 
Phys. Rev. Lett. 69, 3314 (1992).

${ }^{3}$ V. Savona, L.C. Andreani, P. Schwendimann, and A. Quattropani, Solid State Commun. 93, 733 (1995).

${ }^{4}$ R. P. Stanley, R. Houdré, C. Weisbuch, U. Osterle, and M. Ilegems, Phys. Rev. B 5310995 (1996).

${ }^{5}$ R. Houdré, C. Weisbuch, R. P. Stanley, U. Oesterle, P. Pellandini, and M. Ilegems, Phys. Rev. Lett. 73, 2043 (1994).

${ }^{6}$ I. W. Tao, J. K. Son, C. Pecharromán, E. E. Mendez, and R. Ruf, Physica E 2, 685 (1998).

${ }^{7}$ A. R. Pratt, T. Takamori, and T. Kamijoh, Phys. Rev. B. 58, 9656 (1998).

${ }^{8}$ R. Houdré, R. P. Stanley, U. Oesterle, M. Ilegems, and C. Weisbuch, Phys. Rev. B. 49, 23 (1994).

${ }^{9}$ L. S. Dang, D. Heger, R. André, F. Boeuf, and R. Romestain, Phys. Rev. Lett. 813920 (1998).

${ }^{10}$ F. Boeuf, R. André, R. Romestain, L. Dang, E. Peronne, J. F. Lampin, D. Hulin, A. Alexandrou, Phys. Stat. Sol. A 178, 129 (2000).

${ }^{11}$ T. R. Nelson, et al., Appl. Phys. Lett. 69, 3031 (1996).

12 A. R. Pratt, T. Takamori, and T. Kamijoh, Appl. Phys. Lett. 58, 1869 (1999).

13 J. Bloch, T. Freixanet, J. Y. Marzin, V. Theirry-Mieg, and R. Planel, Appl. Phys. Lett. 731694 (1998).

${ }^{14}$ A. Tredicucci, Y. Chen, V. Pellegrini, and C. Deparis, Appl. Phys. Lett. 662388 (1995).

15 J. H. Dickerson, et al., Bull. Am. Phys. Soc. 46, 1228 (2001).

${ }^{16}$ G. H. Wannier, Phys. Rev. 117, 432 (1960).

${ }^{17}$ E. E. Mendez, F. Agulló-Rueda, and J.M. Hong, Phys. Rev. Lett. 602426 (1988).

18 The difference between $E 1_{h h}$ and $E 1_{l h}$ is $16 \mathrm{meV} . m_{h h}=$ $.34 m_{e} \cdot m_{h h}=.094 m_{e}$. Valence band barriers of $138 \mathrm{meV}$ and $193 \mathrm{meV}$ for $\mathrm{x}=0.25$ and 0.35 , respectively ( $\mathrm{x}$ is the Al mole fraction). 